\title{
Reducing the Dauer Larva: molecular models of biological phenomena in Caenorhabditis elegans research.
}

\section{ABSTRACT}

\begin{abstract}
One important aspect of biological explanation is detailed causal modeling of particular phenomena in limited experimental background conditions. Recognising this allows a new avenue for intertheoretic reduction to be seen. Reductions in biology are possible, when one fully recognises that a sufficient condition for a reduction in biology is a molecular model of 1) only the demonstrated causal parameters of a biological model and 2) only within a replicable experimental background. These intertheoretic identifications -which are ubiquitous in biology and form the basis of ruthless reductions (Bickle 2003)- are criticised as merely "local" (Sullivan 2009) or "fragmentary" (Schaffner 2006). However, in an instructive case, a biological model is preserved in molecular terms, and a complex biological phenomenon has been successfully reduced. In doing this the molecular model remains valid in a broader range of background conditions and meaningfully unites disparate biological phenomena.
\end{abstract}

\section{Philosophical Background}

Contemporary intertheoretic reduction of biology -as the epistemological project of formally uniting scientific domains with identities, rather than an ontological analysis of mereologically differentiated levels- begins with E. Nagel $(1949,1961)$, develops with K. Schaffner's application of reduction to genetics $(1967,1993)$ and arrives at J. Bickle's ruthless reductionism in neuroscience $(2003 ; 2006)$. It is by this route that the debate has encountered a conceptual narrows, where the successful reduction of anything of broad explanatory value from biology through into molecular language seems methodologically impossible (Schaffner 2006; Sullivan 2009).

Admittedly (Schaffner 2006), initially promising attempts to connect the formal structure of biology, specifically, the laws of classical genetics with those of molecular genetics, failed to find the required common axiomatisation, even with significant restrictions and restructuring. But on an account of explanation that includes experimental models (e.g. : Cartwright 1983; Hacking 1983; Woodward 2003) -where individual models, not entire theories, do the explaining- reduction requires mapping between particular biological and molecular phenomena. Such one-to-one maps connecting some classically defined gene with a chemically individuated segment of DNA were to provide the necessary and sufficient molecular mechanisms for the biologically individuated phenomenon. Problematically, such maps appear to reveal many-to-many relations between molecular and biological models (Hull 1974). Molecular models exhibit context sensitivity: the 
mechanisms are individuated in terms of differences in their chemical kinds, but as parts of biological wholes one set of chemical cascades can play multiple distinct functional roles in different cellular contexts. Symmetrically, phenomena described by one broadly explanatory biological model (paradigmatically: of eyes or wings) can be instantiated in a gerrymandered group of molecular mechanisms and are thus multiply realised in the chemistry.

Like trying to navigate between Scylla and Charybdis, attempts to identify biological phenomena with molecular mechanism find themselves confronted either by context dependence or multiple realisability. For instance, this is seen in the literature clearly as well understood hurdles to unequivocally identifying the molecular mechanism of the electrophysiological phenomenon of late-phase long term potentiation in neurons (L-LTP) (Bickle 2003, 2006). Beginning with a molecular model requiring a specific "gene expression and protein synthesis" (Bickle 2006 p419) pathway that seems promising as the necessary mechanism of L-LTP, the same pathway ubiquitously re-appears in other cells engaged in biological functions completely unrelated to LTP. But if we start with a model that is broad enough to range over of all phenomena identifiable as LLTP and search for molecular mechanisms, we encounter multiple distinct biochemical pathways responsible for the electrophysiological differences (Malenka and Bear 2004). Such difficulties in mapping intertheoretic identities have recently re-solidified the anti-reductionist consensus in the philosophy of biology despite recent enthusiasm for reductions in neuroscience.

In response, this paper argues that there is a space between context dependence and multiple realisability where numerous intertheoretic identities are already exposed in molecular models of experimentally well-characterised biological phenomena. Simple models of necessary and sufficient molecular mechanisms of biologically identified phenomena are ubiquitous throughout the life sciences but, due to their methodologically precise investigating focus, are seen as disconnected fragments incapable of formally unifying anything of explanatory significance (Schaffner 2006; Sullivan 2009). These models are central to explanation in biology and have been scrutinised philosophically, forming the experimental core of ruthless reductions in neuroscience (Bickle 2003), but still remain unrecognised in the literature for their direct relevance to intertheoretic reductions (e.g.: Wimsatt 1976; Weber 2005; Schaffner 2006; Bechtel 2006; Craver 2007; Sullivan 2009). Accordingly, this paper aims to expose the mechanisms of intertheoretic reduction at work here by focusing on the recently modeled molecular mechanisms of a complex biological phenomenon, an example of sensory-coupled, environmentally-regulated developmental plasticity known as dauer arrest seen in the model organism Caenorhabditis elegans. 
Models such as this one constitute the main points of interaction between chemistry and biology and are, themselves, the important identities that locate the causal mechanisms of a biological phenomenon within a space of molecular differences. What makes these identities justified lies with the fact that they are based on experiments which directly demonstrate the dependency of the biological phenomenon on identified molecular differences. By limiting the intertheoretic identity to the precise model of the observed biological effect within the confines of the controlled laboratory conditions the problems of context dependence and multiple realisability are avoided: the molecular model unambiguously identifies causal mechanisms that are both necessary and sufficient to explain the experimentally observed variation in the biological phenomenon.

However, contrary to the explicit arguments of Sullivan (2009) and Schaffner (2006), limiting intertheoretic identities to experimentally observed effects does not inhibit greater explanatory unification. Rather, it is right here, in the experimentalist's lab that reductions occur: the experiments used to characterise some biological phenomenon as a function of carefully quantified manipulations represents the location where both biological and molecular theories meet, where the same reliable and replicable experimental result is included and explanatorily salient within both theoretical domains. This is how theories in the life science interact. By focusing on preserving the causal explanations resulting from controlled experiments biological phenomena get into the language of chemistry, as models of the relevant molecular mechanisms. Molecular models of biological phenomena can, being now governed by the laws of chemistry, bind to each other in terms of their molecular interactions.

\section{Explanation in the Biological Sciences}

Philosophical treatments of explanation are primarily nomological, where to explain is to have some phenomenon inferred from a set of initial conditions using laws. However, there is scepticism, arising critically in biology, over whether explanations derive their power from the normative force of deduction from laws with universal applicability (Smart 1963; Beatty 1995, Weber 2005). Many philosophers have argued that causal explanations of phenomena within limited backgrounds are, in of themselves, meaningful (Cartwright 1983; Hacking 1981; Woodward 2003). Such causal explanation does not require that the model be universally, or even broadly, applicable, only that the relationship reliably occurs in some specifiable range of circumstances. 
Here, explanatory power lies in the ability to specify the causally relevant differences behind variation in certain measureable quantities. To re-phrase Woodward's (2003) influential formulation, to explain is to ask a set of 'what happens to this something if these conditions had been made a little bit different within this stable background' questions with answers specifying why this something occurred in terms of differences these antecedent conditions.

This matches up with the explanatory practices throughout experimental biology (Weber 2005). A simple laboratory experiment explains an observed effect with a basic causal model. It is through manipulating, or intervening, to introduce a difference into the experimental conditions (a hypothesised cause, i.e.: an independent variable (IV)) and measuring change in this something (the modeled effect, i.e.: a dependent variable (DV)) that the measured biological variation is modeled as the effect of the manipulations. The explanation for the differences between the two values of the DV is thereby interpreted as a causal explanation: the difference in the IV between the manipulated and the control condition is explanatory by being a sufficient cause of the observed difference in the DV. Within laboratory conditions, experiments using standardised populations of model organisms can, and do produce causal models of observed variation in biological traits or behaviours that allow significant intertheoretic identities to be formed. As will be argued, such simple identities, connecting two distinct theoretical explanations, unify.

\section{Molecular Reductions}

Though the perspectives are varied, the vast majority philosophers reject intertheoretic unification through reductive identities. Some prominent philosophers of biology (e.g.: Oyama 1985 and Dupre 1993) have argued that explanations in biology consist of multiple compatible but autonomous theories. This strategy clearly prevents the elimination of biology through a reduction; but pluralism does not unify science. Many pluralists have concluded that some unity is derived from different models overlapping at certain points. Through the interaction of distinct theories at these intersections, multiple disciplines or fields are integrated in order to produce unified explanations (Maull 1977; Mitchell 2003; Schaffner 2006; Brigandt 2010). A number of researchers take this integrative approach more directly and propose unified mechanistic models that

incorporate several ontological levels of theoretically independent description into one explanatory model of mechanisms at multiple mereologically integrated levels (e.g.: Machamer Darden and Craver 2000, Bechtel 2006, Craver 2007). Only a very few see reductions as a legitimate possibility 
(e.g. Bickle 2003, 2006), and these are criticised as being, at best, patchy and isolated models of restricted scope and explanatory value (e.g.: Schaffner 2006; Sullivan 2009).

However, we argue against this current: significant intertheoretic identities are revealed by narrowing our analytic focus on the fine grained details of particular experimental models and away from broad theoretical explanations. It is the widely replicable, well-controlled laboratory experiments revealing invariant causal relationships in standard model organisms that serve as the fulcrum of reductions in biology. This is where both biology and chemistry refer to the same experimental phenomena with casual models employing distinct theoretical resources in their respective explanations. So a molecular reduction (as we will refer to it here) is the formation of a molecular model that experimentally explains the variation in the relevant parameters of a biological model in terms of effective molecular causes of the biological variation within the same experimental background.

It is by this last condition that these reductions are clearly distinguished from ruthless reductions (Bickle 2003, 2006): while both take the same experimental methods as the mechanisms of intertheoretic reduction, molecular reductions remain focused on the experiment itself. This is to say that in molecular reductions the point of identity between the two theories is the experimental effect, the reliable causal relationship demonstrable in a replicable experiment. In this way, molecular reductions are limited in scope to identifying molecular mechanisms of the measured experimental effects, not the theoretical phenomenon they are methodologically assumed to represent. And it is directly by remaining focused on the experimental results that such reductions can avoid the methodological problems ruthless reductions have in extending identities beyond the confines of the experimental conditions.

Bickle argues that research investigating the molecular mechanisms of L-LTP in the mammalian hippocampus is an example of the actual practice of ruthless reductionism in neuroscience. Experiments where the IV and DV are individuated by distinct theories, using what Bickle describes as a methodology of "intervene molecularly and track behaviourally" (2006 p420), explain by causally modeling a biological effect in terms of a molecular variation. Explanation of the electrophysiological phenomenon of L- LTP induction is thereby in terms of the experimentally demonstrated "links" between L-LTP and certain specific molecular mechanisms: a "structural change in the molecular make-up of post-synaptic dendritic spine" (Bickle 2006 p419), as a result of gene transcription under the control of a specific isoform of cyclic AMP response element binding 
protein (CREB). These intertheoretic links are intended to remain explanatory outside the laboratory, to phenomena in the world (all cases of induction of mammalian hippocampal L-LTP) where the justified inference from the experimental results is that these same mechanisms (specific CREB isoform pathway) are responsible for the biological phenomenon seen outside the lab (Bickle 2003 p99).

As many others have argued (de Jong and Schouten 2005; Aizawa 2007; Schaffner 2006; Sullivan 2009) the attempt to map all cases of LTP to a single molecular model is trying to bridge too much across too far a span. They accept that identifying the molecular mechanisms of L-LTP and a limited, local reduction- is possible within the highly controlled conditions of any particular experimental protocol, but that the experiments justify a claimed reduction of the biologically individuated phenomena outside of the specific experimental contexts is something they challenge directly on methodological grounds. The methodological challenge is concise: maximising reliability can come at the expense of external validity. The very effort to make an individual experimental protocol powerful enough to detect a specific effect regularly can impair successful reference to the very phenomenon that the experiments are explaining outside of the lab. Directly, it is by controlling (and changing, e.g.: Waters 2006) the background variation seen in the natural world that the experiment can fail to be representative of the entire biological population. Sullivan explains how experimental protocols will be the result of "differential activity of the constraints of reliability and validity - as determined by investigative aims and interests of the individual researcher or laboratory" (Sullivan 2009 536). Experimental protocols designed to be powerful enough to detect a specific molecular effect may be reliable but can suffer from limited external biological validity.

In the case of in vitro LTP research, in order to construct experimental protocols that can detect variation in specific molecular signals, the researchers control and cancel out much of the biological noise. When biological variation is controlled to get a reliable protocol, the results are not directly representative of the intact organism in natural environmental conditions. Increasing an experiment's reliability through by increasing control over variation within the background conditions makes it biologically artificial. This limits the experimentally justified inferences to situations within the same constrained background. Thereby, as Sullivan argues, Bickle cannot generalise and make identities beyond the lab: there are no methodological grounds for concluding that the identified molecular mechanisms found in the test tube will function the same way out in the world. 
The consequences of the lack of validity appear as demonstrable counterexamples to any broader intertheoretic identity, where the particular molecular mechanisms are neither necessary nor sufficient for L-LTP, making it multiply realised (Bregant, Stožer and Cerkvenik 2010). We find, as a result, that there is an "Embarrassment of Riches" (Malenka and Bear 2004) when it comes to finding multiple distinct molecular mechanisms for the induction of L-LTP, and competing hypotheses for the mechanisms at the same neural synapses in the mammalian hippocampus (Lisman 2003). This leaves only highly-local reductions, those relations between manipulation and effect that have only been demonstrated in the lab. Schaffner explains that such reductions, like the disembodied grin of the Cheshire cat, are "patchy and fragmentary" (2006 p378), working only within very limited conditions and in need of integration. Extending the metaphor, the body of biology sinks below observable levels when an experiment increases its reliability so as to isolate only the molecular differences responsible for an effect. Such experimental control identifies molecular differences that are specific to the particular experimental effect and thereby cannot serve as a general bridge to the broader range of biologically similar phenomena. However, studying just the grin may itself guide our inquiries to the point of valid interaction.

\section{Preserving and expanding upon well-designed experiments}

Diverging from traditional accounts, we argue that through reference to the same precisely quantified replicable experimental phenomena disparate theories can, and do in important areas of biological research, identify common elements. This is where molecular models can avoid context dependence and multiple realisability and where valid inter-theoretic inferences can occur. What makes these reductions philosophically distinctive is that, paradoxically, preserving the explanatory models from experiments within highly controlled, and thereby biologically artificial conditions are the means by which the ends of more general and encompassing reductions in biology are achieved. As is argued, it is the strictly controlled background of a population of homogenous model organisms in highly standardised conditions that actually facilitates the explanatory unification of intertheoretic reductions. It is this control that makes these epistemically important reductions in that both biological and molecular theories include explanations of the identical experimental phenomena, the same experimental regularities. This ability of two theories to formulate models of the very same phenomenon constitutes the point of direct contact between the theories and the locus of valid intertheoretic inferences. 
What makes these identities instructive for discussion of intertheoretic reductions is that they provide greater systematisation of the biological phenomena with a unified molecular model. This completes the requirements for reduction by including separate biological phenomena into the explanatory scope of a single molecular model. While this does not resemble most philosophical accounts of reduction, it does, as is being argued, achieve its aims: a valid inference connecting independent terms in distinct domains (though employing a different mechanism).

The origins of this account of inter-theoretic reduction can be traced back to Kemeny and Oppenheim's 1956 criticisms of contemporary reductionism and shares with it a similar goal: to show that "previous definitions were too narrow in that they excluded most actual cases of reduction."(1956 p17). On Kemeny and Oppenheim's definition, reduction is a replacement of one theory by another theory, but one that preserves the observational vocabulary and leads to equivalent, or greater, theoretical systematisation of the observations. This crucially recognises that in the progress of science, preserving the older theory by connecting its laws and generalisations to the new theory via bridge principles is unnecessary: laws systematise the observations but do not constitute the empirical checkpoints that need to be preserved. For Kemeny and Oppenheim, preservation of the observations across an advance in theory, that is, a change that increases the systematisation of the observations, is both necessary and sufficient for a reduction.

Though a fundamental distinction between theoretical and observational vocabularies is no longer acceptable and a hypothesis cannot be falsified convincingly in isolation of others, simple causal hypotheses tested within replicable experiments can be meaningfully dissociated from their broader theoretical motivations. This allows multiple theories to refer to the identical phenomena. It is the "reliability of experimental knowledge" (Mayo 1996 110) and the theory-independent reality of experimentally demonstrated causal phenomena (Cartwright 1983) that allow the results of a replicable experiment to be included in multiple theories. So, retaining the experimental relationships that were of significance to biology within molecular models makes the causal structure of the biological phenomenon that which is preserved in a reduction. And this is exactly what we want preserved across distinct theoretical explanations and carried through progress in the empirical sciences: those causally effective relations that have been repeatedly demonstrated experimentally. Molecular reduction saves the biological phenomenon as a genuine explanandum of a distinct molecular model. 
But where the biological model of the experimental phenomenon is representative of only normal populations, molecular models are able to quantify and directly incorporate more experimental results than the biological models. In doing this, molecular models reduce biology: unifying multiple biological phenomena by identifying them with elements of one molecular model. And, through this unification, molecular reductions show themselves not to be that patchy in the end. Molecular models can directly identify the molecular mechanisms behind highly complex biological phenomena. This is achieved in dauer arrest in C. elegans. And because the normal population of experimental organisms is only a small subset of the overall background conditions in which such identified molecular mechanisms occur, these (and molecularly similar) signalling pathways are already studied as chemically defined interactions in a wide range of background conditions: in other organisms and in the test tube. Thereby, the explanatory scope of the molecular model is much broader than the biological one, so when these mechanisms are found in different backgrounds, the molecular model does not break down: the scope of the molecular model is derivative of the breadth of the molecular generalisations and the chemically normal conditions in which those generalisations hold. We can now see how this works.

\section{Caenorhabditis elegans: the worm}

The model organism Caenorhabditis elegans (C. elegans), was chosen as a model organism in the 1970s (Brenner 1974), and is now ubiquitous in biology labs where it is used in molecular genetics, developmental biology and recently, behavioural neuroscience. The worm, as it is known, is, arguably, the most comprehensively understood model animal in biological research. An easily housed organism, reproducing hermaphroditically in a three day lifecycle through four larval stages, large numbers of genetically homogenous animals (eg: N2 Bristol strain) are cultured in highly standardised conditions: Nematode Growth Medium (NGM) agar Petri dishes at 15-25 degrees C with worms fed Escherichia coli (Brenner 1974). This control over environment along with invariant development (e.g. determinate cell fates and limited post-transcriptional modification of proteins) makes $C$. elegans an ideal organism for experimental studies needing the stability of large numbers of very similar subjects in replicable experimental conditions.

It is one particular developmental phenomenon that offers an illuminating example of molecular reductions. C. elegans, in response to changes in environmental conditions, will enter a specialised larval state called dauer arrest (Cassada and Russell 1975). This is an alternative, nonobligatory larval stage wherein, if the environmental conditions become reproductively 
unfavourable for the animal, it undergoes a profound morphological, behavioural and metabolic change. The dauer phenotype differs from that of reproductive development observably and unambiguously and these changes are accompanied by the worm acquiring the ability to endure harsh environmental conditions and persist in this state, extending lifespan months beyond a normal 14-21 days. When returned to more favourable conditions, worms recover and continue reproductive development.

Dauer arrest is a quantifiable and measureable difference between two possible larval stages: a bimorphism and an example of phenotypic plasticity wherein multiple phenotypes are expressed by one genotype. Arresting as a dauer larva is one of two possible developmental trajectories during the worm's early larval development, the other leads to a phenotype of rapid maturation and reproduction. It is thereby a true polyphenism of gross anatomical and cellular morphology: arresting is not a simple retardation of normal development but a clear example of epigenetic control over development. Prior to the first larval moult, when, dependent upon differences in external conditions during early larval development, a commitment to develop along one of these two paths begins. The process of arrest is a complex sensory integration of three environmental signals during the first and second larval stages with critical points at the first and second moults; environmental conditions after each moult begins do not alter the developmental trajectory and no intermediate cases of arrest are observed in wild-type worms (Cassada and Russell 1975; Fielenbach and Antebi 2008).

Dauer-like larval stages are major features in the dispersal and reproductive strategies of nematodes, where having biologically appropriate responses to changing environmental conditions is how animals deal with a variable world. The induction of developmental changes in response to contingent conditions is how many species of nematodes survive the unfavourable times or disperse through the environment (Viney 2009). In the genus Caenorhabditis, evolutionary explanation for why there is a period of arrest include (among others) that it is as an adaptation to the cyclic nature of their food source. Importantly, dauer arrest is composed of two independent phenomena: the induction of arrest as a response to environmental influences and the expression of phenotypic differences between dauer and reproductive development. It is this period of induction and a causal model of the process of commitment to development as dauer that has been the focus of detailed research that is of interest to this reductive project. 


\section{Characterising Induction of dauer}

Donald Riddle (1977) began a series of basic exploratory experiments to quantifiably characterise the induction of dauer arrest in the N2 Bristol strain as a response to contingent environmental conditions. Manipulating different parameters of standard culturing conditions, his lab uncovered three independent but interacting parameters that induced dauer arrest: population density, food levels and temperature (Golden and Riddle, 1982; 1984a; 1984b). In addition to the differences to what was varied in the protocols, when manipulations occurred had dramatic effects on induction. Induction has two critical points at each of the first two moults when bifurcations of developmental paths take place that determine whether or not worms arrest. Prior to the first larval moult, if crowding, food and temperature remain within a range, worms irreversibly commit to reproductive development through a second L2 larval stage. However if conditions exceed certain limits based on all three parameters, worms enter a distinguishable pre-dauer L2d. At this stage, the worm changes observably but has not yet irreversibly entered dauer arrest. So, if the worms are transferred to standard culturing plates in sufficient time before the second moult, the worms will moult to the regular L3 stage. Otherwise, populations of worms arrest at rates dependent on strengths of the interacting environmental signals. What emerged from this were reliable empirical models showing changes in the rates of dauer arrest were causally dependent upon (could be increased or decreased) specific quantified and differentially timed changes in environmental conditions (Golden and Riddle, 1984a; 1984b).

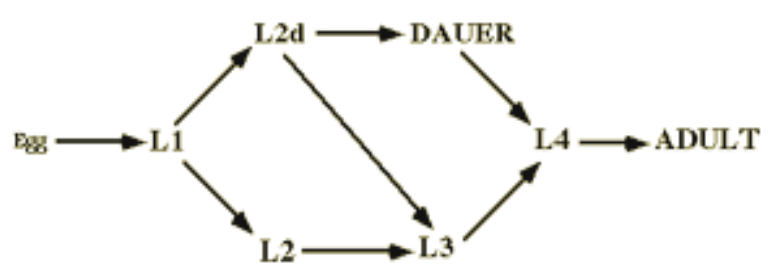

FIG 1: the model of dauer arrest (from D Riddle Lab)

So, by manipulating culturing conditions prior to two bifurcation points (L1 and L2d represented in FIG 1), worms could be reliably manipulated into arresting as dauer. And despite the extensive laboratory research using worms (and comprehensive reporting of results), only four independent manipulations to culturing conditions have been reported to have any significant effect on varying the rates of induction of dauer arrest (WormBase.org Phenotype 0001539):
a) food level
(WBPhenotype:0000148) 

b) cholesterol level
(WBPhenotype:0001847)
(discussed below)
c) temperature
(WBPhenotype:0000639)
d) pheromone level
(WBPhenotype:0000132)

(WormBase.org Phenotype Index 2011)

\section{Biologically salient phenomenon}

Differences between each of these four conditions along with the differences to their timing represent biologically salient differences in the environment that cohere with biological (ecologicalevolutionary-developmental) explanations of dauer arrest. Each particular experiment models one environmentally salient observation (overcrowding, starvation, cholesterol deprivation or heat stress) and connects it to the salient developmental response by the organism: induction of arrest as dauer that permits reproductive survival through such harsh environments. Thus, each individual condition has a good ecological and evolutionary explanations citing response to ecological pressures and subsequent differential rates of survival based on a chosen developmental path. The relevance of these experiments is as evidence integrated into broader theoretical explanations. Additionally, though dauer induction is primarily a developmental phenomenon its relevance extends to explanations in ecology, evolutionary, and neuroscience, and can be seen as a relevant case study in the analysis of research in the emerging field of eco-evo-devo and how we understand the relations between its component disciplines' models (e.g. : Hall and Hallgrímsson 2008; Gilbert and Epel 2008).

\section{The experimental manipulations: the dauer assays}

While research on dauer induction has wide ranging theoretical influence, it is, however 1) the causal regularities found in 2) the highly controlled, replicable conditions of the experimental protocols that forms the philosophically interesting point of intertheoretic identity. These protocols produce easily replicable conditions in which dauer arrest is reliably induced. These form the stable fulcrum of reduction that remains unaffected by conceptual changes, or differences in disparate and seemingly incommensurate theories. 
Each of the four independent manipulations $(\boldsymbol{a}-\boldsymbol{d})$ is a simple variation of the standard cultures (Brenner 1974) and consists of one manipulation that increases rates of arrest: these are known as dauer assays. They are also 2) the experimental background conditions in which 1) the causal regularities are found.

In the standard cultures, the absence of arrest is expected; normal worms do not arrest without one of the conditions a-d falling outside a range. However, $\boldsymbol{a}$ ) conditions of food deprivation reliably induce dauer arrest at rates significantly above controls in standard cultures. It is the lack of food that directly explains arrest in this situation and is 2) a specific experimental protocol that can be repeated and where 1) this reliable regularity can be observed. However, $\boldsymbol{b}$ ) only removing cholesterol from the standard culturing plates (which is a component of NGM) also induces arrest, albeit at low but significant frequencies, but does so in the presence of food (Gerisch et al., 2001). These two distinct phenomena with independent explanations for why there was a difference in observed rates of arrest. In one, the difference in food conditions $(+/-e$ coli) between the intervention and control conditions explains differences in rates of arrest, while in the other it is a distinct set of conditions (+/- cholesterol) that causally explains why there is a difference when contrasted with control conditions.

In $\boldsymbol{c}$ ) the third treatment, only temperature of the standard cultures was changed: worms will arrest at much higher rates than controls (in 15-25 C) when exposed to $27 \mathrm{C}$ temperatures (Ailion and Thomas, 2000). In $\boldsymbol{d}$ ) the final of the four conditions, the only difference is to the levels of exogenous daumone -a chemically well-characterised molecule that is produced by worms (Butcher et al 2009) - is added. During the early characterising experiments, D. Riddle discovered that a given level of additional daumone would induce arrest as a function of food available in the environment and that raising levels above $25 \mu \mathrm{l} / 2 \mathrm{ml} \mathrm{NGM}$ at $20 \mathrm{C}$ reliably induced dauer at rates of $100 \%$, even in the presence of abundant food (Golden and Riddle, 1984b).

It is this set of repeatable replicable experimental protocols that form the basis of the tractable interaction between biological and molecular theories. The regularities seen in these dauer assays can be referred to by both the biological as well as the molecular theories, each drawing their own theoretical implications from the very same replicable phenomena. How a direct intertheoretic link is established is seen by the fact that the manipulated conditions in the biological experiment (the dauer assays) have become the controls in the molecular ones. This is the identical 
experimental relationship in both molecular and biological theories that is identified in a molecular reduction.

\section{Disentangling the biological phenomenon from the biological theory}

Now, even though biological theories were used in designing the experiment and modelling the hypotheses tested, replicable experiments justify our inference that the experimental phenomena exist independently of the biological explanations. In reproducing one of the experiments, e.g. culturing in high daumone levels, the expectation would be to observe high rates of arrest. Why this is expected is based on previous experiments demonstrating this regularity in these same controlled conditions. What allows such an expectation in this situation is the phenomenological generalisation which would be reliable and predictive, even if one remained wholly ignorant of the biological explanations (or if they were, perhaps, incorrect). This is what replicable experiments provide: a reasonable expectation about what would happen in the world under certain conditions, expectations that are not dependent upon broad theoretical commitments or assumptions but only on local causal regularities discovered in limited contexts.

It is replicability that allows distinct theories to model and explain the same regularities. Accordingly, in conditions of high pheromone (where arrest is guaranteed) or on the standard plates (where dauer is exceedingly rare) finding that an intervention (e.g.: of the worms' physiological structure or genetics) changes the expected rates of arrest, one can conclude that this difference causally explains the differences in rates of arrest. By manipulating an experimental treatment group in these controlled conditions one contrasts the rates of response with those in the control group; but the control group is just the regularity of response that is explained biologically. So the justification for the claim that both biological and molecular theories are referring to the same phenomenon is that the molecular experiments are occurring in the identical conditions. This fact forms the intertheoretic identity -it is the same reliable, repeatable phenomenon that was identified in the biological experiments that is being modeled and explained with molecular interventions. As argued for in Bickle (2003), with such experimental methods it is the molecular manipulation that explains the variation in the biological trait. However, unlike in Bickle's model, it is only the measured variation seen in the experimental background that is identified, not the theoretically entangled biological or psychological phenomena. 


\section{The molecular model}

The past three decades of research on the induction of dauer arrest has produced "a unified model for dauer formation whereby graded signals from the environment regulate a hormonedependent switch" (Fielenbach and Antebi 2008 pg 2157). This switch is a physiologically widely expressed nuclear hormone receptor complex (DAF-12 NHR) that controls development through controlling gene expression. In the absence of its naturally occurring ligand, the hormone dafachronic acid (DA), DAF-12 NHR, interacting with heterochronic genetic signalling, binds with a co-repressor (DIN-1) to deactivate reproductive development and activate a sequence of specific dauer programs.

The regulation of DAF-12 NHR through critically timed changes to levels of DA is the regulation of arrest: DAF-12 remaining unliganded by DA is required for dauer formation (Magner and Antebi 2008). However, while the regulation of DAF-12 NHR is considered to be sufficient (in normal worms) and necessary (in all known experimental conditions) for arresting in C. elegans (Magner and Antebi 2008), to reduce the model of dauer induction requires the molecular modeling of the environmental regulation of the NHR, not just identification of the switch itself.

While the regulation of DAF-12 is the regulation of dauer arrest, the receptor itself is only regulated through DA at critical points to induce dauer at specific times. So it is insufficient to cite only DA differentially binding to DAF-12 as the mechanism of dauer arrest; it is also necessary to include the relevant regulatory mechanisms to avoid the problem of context dependence. This includes both a molecular model of the regulation of DAF-12 by DA -namely the regulation of the timing of arrest based on the difference in the timing of DA bonding with DAF-12-, and the regulation of DA production- its regulation by molecular signalling pathways connected to environmental inputs. This means that the model of arrest must complete the connection of DAF-12 regulation, through the regulation of DA by signalling pathways that connect back to variation in each of the four environmental conditions that induce arrest

Arresting as a dauer involves changes in a complex network of signalling pathways with multiple spatio-temporally defined steps, modulated by many different factors. However, a complex and coherent molecular model of the causal structure of the biological model (the environmental differences -food level, cholesterol level, temperature and pheromone level- and the developmental timing differences at the two crucial points -represented as L1 and L2d bifurcations in FIG 3) has now been formed. 


\section{The basic model of regulation}

The regulation of arrest is a complex molecular model and can only be sketched in its broadest outlines: a very small review for a small worm. In sensory neurons ASI and ADF activity of a guanylyl cyclase pathway (cGMP) is regulated by competing signals from several different classes of G-protein coupled receptor molecules (GPCR) that respond differently to food signals and daumone (Bargmann and Horvitz 1991). These GPCRs are exposed to the external environment inside sensory structures called amphids where they modulate cGMP. cGMP is up-regulated in the presence of food or down-regulated in the presence of daumone. In the ASI and ADF neurons increased activity of cGMP up-regulates the activity of an insulin-like pathway (IIS). In only the ASI neuron, lowered cGMP signalling also suppresses a transforming growth factor- $\beta$ (TGF- $\beta$ )-like pathway. In ADF as well as AIX and AIY interneurons, cGMP and serotonin signalling both decrease with increases of temperature (Ailion and Thomas, 2000). It is in these neurons that these three environmental signals are modulated, the final signal (cholesterol- which is not neural coupled with environmental signals) is integrated with the neural signalling at the next stage, the production of DA in the XXX cells.

The XXX cells are endocrine cells and the location where the DA hormone biosynthesis pathway becomes involved; these cells express DAF-36 and DAF-9: the first and final stages in the synthesis of DA from cholesterol. During early larval development, the two XXX cells remain in physical contact with these amphid neurons which allows for direct signalling from the neurons by the TGF- $\beta$ and IIS pathways. In these cells, membrane bound receptors, DAF-1/DAF-4 and DAF-2, respond to the lowered extracellular TGF- $\beta$ and IIS signalling from the neurons. Lowered IIS signalling stops inhibiting DAF-16/FOXO; reduced TGF- $\beta$ signalling reduces DAF-8/ DAF-14 signalling which inhibits DAF-3/DAF-5. Both DAF-16/FOXO and DAF-3/DAF-5 prevent DA synthesis but are inhibited themselves under conditions of high TGF- $\beta$ and IIS signalling from the neurons.

These signals from the neurons and ultimately from environmental conditions converge on the modulation of DAF-9 levels in XXX cells. DAF-9 completes the process of converting cholesterol into DA. So, due to the fact that $C$ elegans requires cholesterol in its diet, its absence explains why the cholesterol deprivation conditions induce arrest independently of the others. So it is the 
modulation of DAF-9, the final stage in the production of DA, which represents the mechanisms by which integration of these molecular signalling pathways occurs.

Finally, because DA is a steroid hormone (Motola et al 2006) it can diffuse rapidly throughout all the worm's tissues where it can bind to DAF-12 NHR, and temporally coordinate the regulation of gene expression across the entire organism. The coordination involves DAF-12 NHR interaction with the heterochronic genes lin-4, lin-14, lin-28, lin-29 under a regulatory feedback loop controlled by a family of let-7 micro-RNAs (Hammell, Karp and Ambros 2009) to control DAF12NHR. This stage represents the temporal mechanisms of the critical developmental periods (bifurcations in FIG 1). Without DA during the late L1 larval stage, DAF-12, interacting with genes lin-4, Iin-14, will halt the gene expression required for reproductive development. With reproductive genetic programs switched off, the appropriate L3 cell division programs are not initiated -explaining the L2d phenotypic differences- and the worms continue the development along the path to complete arrest unless levels of DA are increased before a critical time. DA is capable of reversing arrest via action of continued activity of the genes lin-28 and lin-29 prior to the point where metabolic activity in the worms almost completely ceases. Herein, commitment to arrest is achieved and the process of dauer induction is complete: if DA levels are raised, worms will no longer recover to activate L3 cell division programs but proceed directly to arrest and recovery as post-dauer L4 (FIG 1).

What has been achieved in the case of dauer arrest is establishing the identity of the molecular differences that explain the observed difference in rates of arrest discovered in the characterising experiments and reliably observed in each of the dauer assays and controls. What this model of its regulation does is disambiguates the function of DAF-12 in distinct biological contexts. The inability to identify DAF-12 NHR as the molecular mechanism of the experimentally observed rates of arrest was due to the fact that it was regulated by additional environmental and developmental factors. By including the regulatory mechanisms of DAF-12, from DA all the way back to variation in the different environmental conditions during developmental stages -all within a single molecular model- allows a route from defined molecular variation to be mapped directly onto the biological phenomenon: the regulation of this network is necessary and sufficient for the induction of dauer arrest. So here we have the answer to the challenge from context dependence: quantifying the relevant context disambiguates functions. It is the differential activity of complex regulatory networks that form the molecular mechanisms that are identified with the biological phenomenon of induction of dauer arrest. We can now try to analyse this claim. 


\section{The Philosophical Manipulation}

Molecular reductions are different from many traditional approaches to intertheoretic relations as they begin with an examination of the experimental results. However in this way they are much like many recent analyses of reduction and explanatory unification (Bickle 2003; Mitchell 2003; Bechtel 2006, Weber 2005, Schaffner 2006; Sullivan 2009; Brigandt 2010). But by focusing on intertheoretic identities in the lab they are clearly distinguishable from both recent integrative models of unification and ruthless reductions. However, despite the fact that the intertheoretic identities between biology and chemistry are limited to causal relations in experimental conditions, they remain significant reductions.

Like ruthless reductions, molecular reductions unify by modeling the effective experimental manipulations of a biological effect at a single molecular level and, thereby, reduction is achieved through intertheoretic identity. Alternatively, many philosophers look at these same kinds of experiments and describe mechanistic explanations (Wimsatt 1976; Machamer, Darden and Craver 2000), or strategies of localisation and decomposition (Bechtel and Richardson 1993; Bechtel 2006). The philosophical accounts they offer explicate how models at the molecular level are integrated into explanations of biological phenomena. These are not intertheoretic identities and remain broadly pluralist in orientation in so far as integration is characterised by the use of multiple theories in one explans.

Though highly heterogeneous, integrative explanations in biology generally involve elements from many distinct theories that form a single coherent explanation of how some biological process takes place (Maull 1977; Mitchell 2003, Bechtel 2006; Schaffner 2006; Craver 2007; Brigandt 2010). In this way integrative explanations are composites that include everything from mathematical models and computational simulations to experimentally demonstrated and well-quantified effects. These are combined into one single model where each element contributes to the explanation as part of the explanans for some biological explanadum. It is the use of multiple theoretical levels integrated together into one explanans that unifies and demonstrates that there is significant intertheoretic communication and exchange of information between the disciplines; this is also the reason why these are pluralist positions that do not establish intertheoretic identities.

In this regard, molecular reductions are not multi-level explanations; they provide an exclusively molecular explanans for a biological explanandum. For example, spatially localising the production of DA to the XXX cells does not constitute a physiological level element in the explanans. 
The physiological differences (presence or absence of cell) are not the explanatory element of the model, the cell is merely the locus of the causally relevant molecular differences. A physiological manipulation, ablating cells, may change the gross anatomy, but nonetheless, the physiological changes are only the means to achieve specific molecular changes (i.e.: removing hormone production -farm and lab animals are castrated for the same reason). Ablating a cell removes its particular molecular properties from the system, and identifying the molecular difference models the causal factor in terms of molecular-level differences. This is not a multi-level explanation but an explanation based on hypothesised and experimentally tested molecular differences, not gross physiology. So ablating the XXX cells alters the molecular conditions and the relevant difference is a drastic reduction in DA levels (Ohkura et al., 2003). It is this molecular difference that explains changes in arrest, not the physiological difference. Correspondingly, the molecular model of dauer only explains why there is a difference in rates of arrest between the experimental and control groups within these replicable conditions. The specific explanandum is the differential rates of arrest as seen in the laboratory within the dauer assays. In this way the worm's physiological structures and developmental differences are not part of the explanandum but the controlled background conditions for investigating the mechanisms for the induction of dauer arrest.

And so it is by being only the causal mechanisms behind the experimental effect that is identified with the molecular model, molecular reductions avoid the kinds of problems with multiply realised biological phenomena (e.g.: L-LTP) that remain a problem for ruthless reductions. The phenomenon identified herein is the rate of induction of dauer arrest, unequivocally identified with both physiological measures and chemical tests for arrest; the experiments all explain a single effect with the same measurements. That this is the same explanandum in both biological and the molecular models is transparent in so far as the experiments measured the same effect the same way. Quite directly, what is being explained by both theories' models is the effect as observed in the test tube (Petri dish or Erlenmeyer flask to be precise).

So we can see that the molecular model adequately identifies the necessary and sufficient causal mechanisms of the specific experimental effect, but one only seen in the lab; it does not explain all biologically salient cases of the induction of dauer in the wild. Nonetheless, contrary to the explicit views of Sullivan (2009) and Shaffner (2006), this is, by itself, an important point of intertheoretic contact, a reduction of a biologically salient phenomenon. A central goal of intertheoretic reduction is to form identities that preserve reference where both theories refer to the same phenomena: preservation of reference with an identity explains how there is meaningful 
connections and communication between scientific domains (reduction as unification, distinguished from autonomy), not to mention any theoretical continuation as opposed to incommensurate change (reduction as progress, distinct from revolution). In the worm, it is the experimental model of arrest (FIG-1) that has been preserved within a molecular model.

What makes the reduction of dauer induction illustrative lies in comprehensiveness of the molecular model: all biologically identified causally effective pathways from the environment that were found in the dauer assays (food level, cholesterol level, temperature and pheromone level together with the timing around two critical points) are represented in the molecular model. By finding the molecular mechanisms of the what, when and where of induction that the molecular model of how worms were induced to arrest as response to the environment was finally traced out from beginning to end. So since this is the identification of the many distinct molecular signalling pathways responsible for a complex and biological significant phenomenon, this is also clearly a reduction that exhibits significant unification of the biological sciences, to which we can finally turn.

\section{The Circe Effect: from biological enthymemes to molecular deductions.}

This reduction unifies by linking all the experimental manipulations into a single model; the main benefit of the reduction arises from the fact that this model remains valid in a broader range of conditions. This is seen directly in the dauer research where experiments in multiple conditions (in the dauer assays) can all use a single molecular model. The wider potential unification of molecular reductions is seen in the role such molecular models play in the molecular biologist's lab in order to explain more biological process with molecular mechanisms and connect a wider range of biological phenomena within the single molecular model.

Practically, explaining variation in rates of dauer arrest through a single molecular model provides a more unified explanation than the biological models. Since the induction of dauer consists of two biologically independent redundant and parallel pathways (a neuro-sensory and a distinct metabolic coupling with the environment -c elegans requires dietary cholesterol), a single molecular model that includes both as elements of one set of regulatory mechanisms of DAF-12 NHR -converging on DAF-9 (see above) is substantively more unified. Thus, the primary importance of this reduction lies in the comprehensiveness of the molecular model which directly demonstrates that a reduction can retain complete models of complex phenomenon from developmental biology (i.e. FIG-1). So extending Schaffner's metaphor, in the case of the induction 
of dauer arrest we may only have a grin left to study, but as is characteristic of Cheshire cats, it is very wide and reveals much.

This is where the biologically limited conditions of highly controlled experimental backgrounds display their full potential for theoretical unification. The worm, being a simple and stable system, forms a set of replicable background conditions in which bio-chemists study and better "understand the nature of biomolecules and the interactions among them" in terms of "molecular phenomena such as molecular recognition, the hydrophobic effect, multivalency, enzymatic catalysis, and signal transduction" (Hulme and Whitesides 2011). Using the worm, researchers can experimentally detect specific chemical differences over the molecular noise of many regulatory networks running at full steam. Methodologically, this makes the worm the test tube in which precise molecular events can be studied against the background of a complex living system. This molecular research exposes more depth and detail to the causal structures of the various phenomena, exposing molecular mechanisms by which disparate models of other biological phenomena can connect to each other: it can bind multiple biologically significant phenomena as elements of one larger molecular network and include more of it as a model of a single interconnected system. This is how greater explanatory unification of biological models takes place.

The final virtue of molecular reductions is thereby revealed as they provide the general means by which unification proceeds: disparate biological phenomena are interconnected in terms of activity of their causally relevant molecular pathways. The ability to see the living system in terms of molecular mechanisms unifies biology by giving us the means by which to conceive of distinct biological processes as processes of one system. Like the metabolic and neural pathways are unified through their convergence as causal pathways on a single molecular mechanisms, we can model more of the underlying molecular mechanics of the many parts of the Rube-Goldberg structure of living systems in terms their unified causal structure, now seen as one interacting mechanism at the molecular level, as a single system of interacting parts. This unity is only revealed at the molecular level where the systematic causal relations between different biological models are exposed.

We can finally see that molecular reductions provide molecular mechanisms for connecting seemingly distinct models. Such points of identity are thereby significant reductions through the quantification of a biologically important model within a molecular space, distilling the causal structure of phenomena to the effective chemical differences. These chemical differences interact as 
a network of biologically identifiable molecular pathways that attach to each other through their chemically governed interactions. Unification, and a molecular reduction is thereby achieved by allowing molecular bonds to form between biologically independent phenomena.

\section{References}

Ailion, M., and Thomas, J.H. (2000). Dauer formation induced by high temperatures in Caenorhabditis elegans. Genetics 156, 1047-1067.

Aizawa, K. (2007). The biochemistry of memory consolidation: A model system for the philosophy of mind. Synthese, 155(1), 65-98.

Bargmann CI, Horvitz HR.Science. 1991 Mar 8;251(4998):1243-6.Control of larval development by chemosensory neurons in Caenorhabditis elegans.

Beatty John, 'The Evolutionary Contingency Thesis' in G. Wolters and J. Lennox (eds.), Concepts, Theories and Rationality in the Biological Sciences (Pittsburgh: University of Pittsburgh Press, 199547

Bechtel, W. (2006). Discovering cell mechanisms: The creation of modern cell biology. Cambridge: Cambridge University Press.

Bechtel, W., \& Richardson, R. C. (1993). Discovering complexity: Decomposition and localization as strategies in scientific research. Princeton: Princeton University Press.

Bickle, J. (2003). Philosophy and neuroscience: A ruthlessly reductive account. Dordrecht: Kluwer Academic Publishing.

Bickle, J. (2006). Reducing mind to molecular pathways: Explicating the reductionism implicit in current cellular and molecular neuroscience. Synthese, 151(3), 411-434.

Brenner S (1974) The genetics of Caenorhabditis elegans. Genetics. 1974 May;77(1):71-94.

Brigandt, I. (2010). Beyond reduction and pluralism: Toward an epistemology of explanatory integration in biology. Erkenntnis, 73, 295-311.,

Bregant, J. Stožer, A. Cerkvenik, M. (2010) Molecular reduction: reality or fiction? Synthese 172:437-450

Cartwright, N. (1983). In Oxford University Press (Ed.), How the laws of physics lie. Oxford; Oxford : New York; Oxford : Clarendon Press, 1983: Oxford : Clarendon Press.

Cassada, R. C., and R. L. Russell, 1975 The dauer larva, a post-embryonic developmental variant of the nematode Caenorhabditis elegans. Dev. Biol. 46: 326-342. 
Craver, C. F. (2007). Explaining the brain: Mechanisms and the mosaic unity of neuroscience. Oxford: Oxford University Press.

De Jong, H. L., and Schouten, M. K. D. (2005). Ruthless reductionism: A review essay of john bickle's philosophy and neuroscience: A ruthlessly reductive account. Philosophical Psychology, 18(4), 473-486.

Dupré, J. (1993). The disorder of things: Metaphysical foundations of the disunity of science. Cambridge, MA: Harvard University Press.

Fielenbach N and Antebi A (2008) C. elegans dauer formation and the molecular basis of plasticity Genes \& Development, Volume: 22, Issue: 16,: 2149-2165

Gerisch, B., and Antebi, A. (2004). Hormonal signals produced by DAF-9/cytochrome P450 regulate C. elegans dauer diapause in response to environmental cues. Development 131, 1765-1776

Gilbert S.F., Epel D. (2008) Ecological Developmental Biology, Sunderland: Sinauer

Golden, J.W., and Riddle, D.L. (1982). A pheromone influences larval development in the nematode Caenorhabditis elegans. Science 218, 578-580.

Golden, J.W., and Riddle, D.L. (1984a). The Caenorhabditis elegans dauer larva:

developmental effects of pheromone, food, and temperature. Dev. Biol. 102, 368-378.

Golden, J.W., and Riddle, D.L. (1984b). A pheromone-induced developmental switch in Caenorhabditis elegans: Temperature-sensitive mutants reveal a wild-type temperaturedependent process. Proc. Natl. Acad. Sci. U.S.A. 81, 819-823.

Hacking, I. (1983). Representing and intervening: Introductory topics in the philosophy of natural science. Cambridge: Cambridge University Press.

Hall, B. K., and Hallgrímsson, B. (2008). Strickberger's Evolution. The Integration of Genes, Organisms and Populations. 4th Edition. 776 pp. Jones and Bartlett

Hull, D. L. (1974). Philosophy of biological science. Englewood Cliffs: Prentice-Hall.

Hammell, C.M., Karp, X. and Ambros, V. (2009) A feedback circuit involving let-7-Family miRNAs and DAF-12 integrates environmental signals and developmental timing in $\mathrm{C}$. elegans. Proc Natl Acad Sci U S A.

Hulme and Whitesides 2011 Chemistry and the Worm: Caenorhabditis elegans as a Platform for Integrating Chemical and Biological Research

John G. Kemeny \& Paul Oppenheim (1956). On Reduction. Philosophical Studies 7 (1-2):6 19.

Kiontke, K. and Sudhaus, W. Ecology of Caenorhabditis species (January 09, 2006), WormBook, ed. The C. elegans Research Community, WormBook, doi/10.1895/wormbook.1.37.1, http://www.wormbook.org. 
Lisman, J. (2003) Long-term potentiation: outstanding questions and attempted synthesis Phil. Trans. R. Soc. Lond. B 358, 829-842

Machamer, P., Darden, L., \& Craver, C. F. (2000). Thinking about mechanisms. Philosophy of Science, 67, 1-25.

Malenka, R and Bear, M. LTP and LTD: Review An Embarrassment of Riches Neuron, Vol. 44, 5-21, September 30, 2004

Magner, D.B., Antebi, A., 2008. Caenorhabditis elegans nuclear receptors: insights into life traits. Trends Endocrinol. Metab. 19, 153-160.

Maull, N. (1977). Unifying science without reduction. Studies in the History and Philosophy of Science, 8, 143-171.

Mayo, D . (1996) Error and the Growth of Experimental Knowledge. Chicago: The University of Chicago Press.

Motola DL, et al. (2006) Identification of ligands for DAF-12 that govern dauer formation and reproduction in C. elegans. Cell 124:1209-1223

Mitchell Sandra (2003) Biological Complexity and Integrative Pluralism, Cambridge: Cambridge University Press.

Nagel, E. (1949)"The Meaning of Reduction in the Natural Sciences," in Science and Civilization, edited by R. C. Stauffer (Madison: University of Wisconsin Press,).

---- (1961). The structure of science; Problems in the logic of scientific explanation. New York: Harcourt.

Ohkura, K., Suzuki, N., Ishihara, T., and Katsura, I. (2003). SDF-9, a protein tyrosine phosphatase-like molecule, regulates the L3/dauer developmental decision through hormonal signaling in C. elegans. Development 130, 3237-3248.

Ogawa A. and R. Sommer, Strategies to Get Arrested Science 13 November 2009: Vol. 26 no. 5955 pp. $944-945$

Oyama S (1985) The ontogeny of information: Developmental systems and evolution. Cambridge: Cambridge University Press.

Riddle, D. L. (1977). A genetic pathway for dauer larva formation in Caaorhabditis elegans. Stadkr Genet. Svmp. 9,101-120.

Riddle DL, MacMorris MA. 1981. Critical periods in the development of the Caenorhabditis elegans dauer larva. Dev Biol 84:27-40.

Schaffner, K. (1967). Approaches To Reduction. Philosophy of Science, 34, 137.

----. (1993a). Discovery and Explanation in Biology and Medicine. Chicago, University of Chicago Press.

----. (2006). Reduction: The cheshire cat problem and a return to roots.151(3) 
Smart, J. J. C. , Philosophy and Scientific Realism (London: Routledge \& Kegan Paul, 1963), 53-54.

Sullivan, J. A. (2009). The Multiplicity of experimental protocols: A challenge to reductionist and non-reductionist models of the unity of science. Synthese, 167, 511-539.

Viney, M.E. (2009) How did parasitic worms evolve? BioEssays, 31, 496-499.

Weber, M. (2005). Philosophy of experimental biology. Cambridge: Cambridge University Press

Wimsatt, W. C. (1976). Reductive explanations: A functional account. In R. S. Cohen \& A. Michalos (Eds.), Proceedings of the 1974 biennial meeting of the Philosophy of Science Association (pp. 671-710). Dordrecht: Reidel.

Woodward, J. (2003). Making things happen: A theory of causal explanation. Oxford: Oxford University Press.;

Michal Arciszewski (Doctoral Candidate)

Dept. Of Philosophy

Univ. Of Guelph

marcisze@uoguelph.ca 